\title{
Weight Loss, Dietary Intake and Pulse Wave Velocity
}

\author{
Kristina Petersen Natalie Blanch Jennifer Keogh Peter Clifton \\ Division of Health Sciences, School of Pharmacy and Medical Science, University of \\ South Australia, Adelaide, S.A., Australia
}

\section{Key Words}

Pulse wave velocity $\cdot$ Arterial stiffness $\cdot$ Weight loss $\cdot$ Diet

\begin{abstract}
We have recently conducted a meta-analysis to determine the effect of weight loss achieved by an energy-restricted diet with or without exercise, anti-obesity drugs or bariatric surgery on pulse wave velocity (PWV) measured at all arterial segments. Twenty studies, including 1,259 participants, showed that modest weight loss ( $8 \%$ of the initial body weight) caused a reduction in PWV measured at all arterial segments. However, due to the poor methodological design of the included studies, the results of this meta-analysis can only be regarded as hypothesis generating and highlight the need for further research in this area. In the future, well-designed randomised controlled trials are required to determine the effect of diet-induced weight loss on PWV and the mechanisms involved. In addition, there is observational evidence that dietary components such as fruit, vegetables, dairy foods, sodium, potassium and fatty acids may be associated with PWV, although evidence from well-designed intervention trials is lacking. In the future, the effect of concurrently improving dietary quality and achieving weight loss should be assessed in randomised controlled trials.
\end{abstract}

(C) 2015 S. Karger AG, Basel

\section{Introduction}

Poor diet, including a high intake of energy-dense, nutrient-poor foods, is a leading cause of ill health in Australia and around the world [1]. Worldwide, the prevalence of obesity has more than doubled since 1980 [2]. Obesity increases the risk of cardiovascular disease (CVD) by approximately two fold [3]. At present, the effect of weight loss on cardiovascular endpoints is not clear [4], and due to the methodological difficulties associated with conducting these types of studies, a large body of research has looked at measures of vascular function that 
Petersen et al.: Weight Loss, Dietary Intake and Pulse Wave Velocity

predict cardiovascular outcomes. We recently conducted a meta-analysis to determine the effect of weight loss achieved with an energy-restricted diet with or without exercise, antiobesity drugs or bariatric surgery on pulse wave velocity (PWV) measured at all arterial segments [5].

Carotid femoral PWV (cfPWV) is considered the gold standard for determining arterial stiffness because the propagation of the forward pressure is measured at the aorta [6]. It is an independent predictor of cardiovascular events and mortality, and addition of cfPWV to the conventional Framingham risk factors improves CVD risk prediction [7]. A systematic review of observational studies found that age and blood pressure [systolic blood pressure: $\mathrm{n}=43(43 \%)$ or mean arterial pressure: $\mathrm{n}=30(31 \%)]$ were independent predictors of cfPWV in 91 and $90 \%$ of all studies, respectively [8]. The systematic review conducted by Cecelja and Chowienczyk [8] concluded that other than age and blood pressure, cfPWV is largely independent of traditional risk factors for CVD. Therefore, it is possible that dietary factors, including diet-induced weight loss and overall dietary intake, could predict PWV.

\section{Weight Loss and PWV}

The meta-analysis of 20 studies, including data from 1,259 participants, showed that modest weight loss (8\% of the initial body weight) caused a reduction in PWV measured at all arterial segments [5] [fig. 1]. Subgroup analysis showed that there was not a statistically significant difference in the change in PWV achieved with an energy-restricted diet alone (10 studies) or an energy-restricted diet plus exercise (8 studies). Meta-regression showed that the change in PWV achieved with weight loss was positively correlated with the change in systolic and diastolic blood pressure. However, from the research that has been conducted it is unclear whether the blood pressure reduction precedes PWV change or is a result of a lowered PWV. Another possible mechanism to explain the effect of weight loss on PWV is a reduction in inflammation. Current evidence suggests that arterial stiffness is largely driven by inflammatory processes that impair endothelial nitric oxide production, generate reactive oxygen species and the release of neutrophils, macrophages and cytokines [9]. It is known that obesity is associated with an increase in inflammatory cytokines [10], and weight loss reduces inflammation [11]. A limitation of the meta-analysis conducted is that we did not extract data on inflammation molecules from the included trials.

This meta-analysis suggests that weight loss may be an effective strategy to reduce arterial stiffening. However, the methodological quality of the studies included was poor, and therefore, well-designed randomised controlled trials are required to determine the effect of weight loss on PWV. In particular, the macro-nutrient composition of the diet needs to be investigated further, as the studies included in this meta-analysis were too heterogeneous with respect to the diet to determine the effect of dietary composition on PWV. Furthermore, future research should aim to determine the mechanism by which weight loss has an effect. The results of this meta-analysis may not be generalizable to certain population groups. For example, only 3 studies (all uncontrolled) included in this meta-analysis were conducted in cohorts with diabetes (all type 2) or impaired fasting glucose. Two of the 3 studies did not achieve a statistically significant reduction in cfPWV with weight loss, although subgroup analysis showed that there was no statistically significant difference in the change in PWV by the diabetes status of the subjects. However, given the small number of studies and the low methodological quality, further research is required to determine whether weight loss is an effective strategy to reduce arterial stiffness in patients with diabetes. This is an important question because subjects with type 2 diabetes have a heightened risk of CVD compared with the general population [12]. 


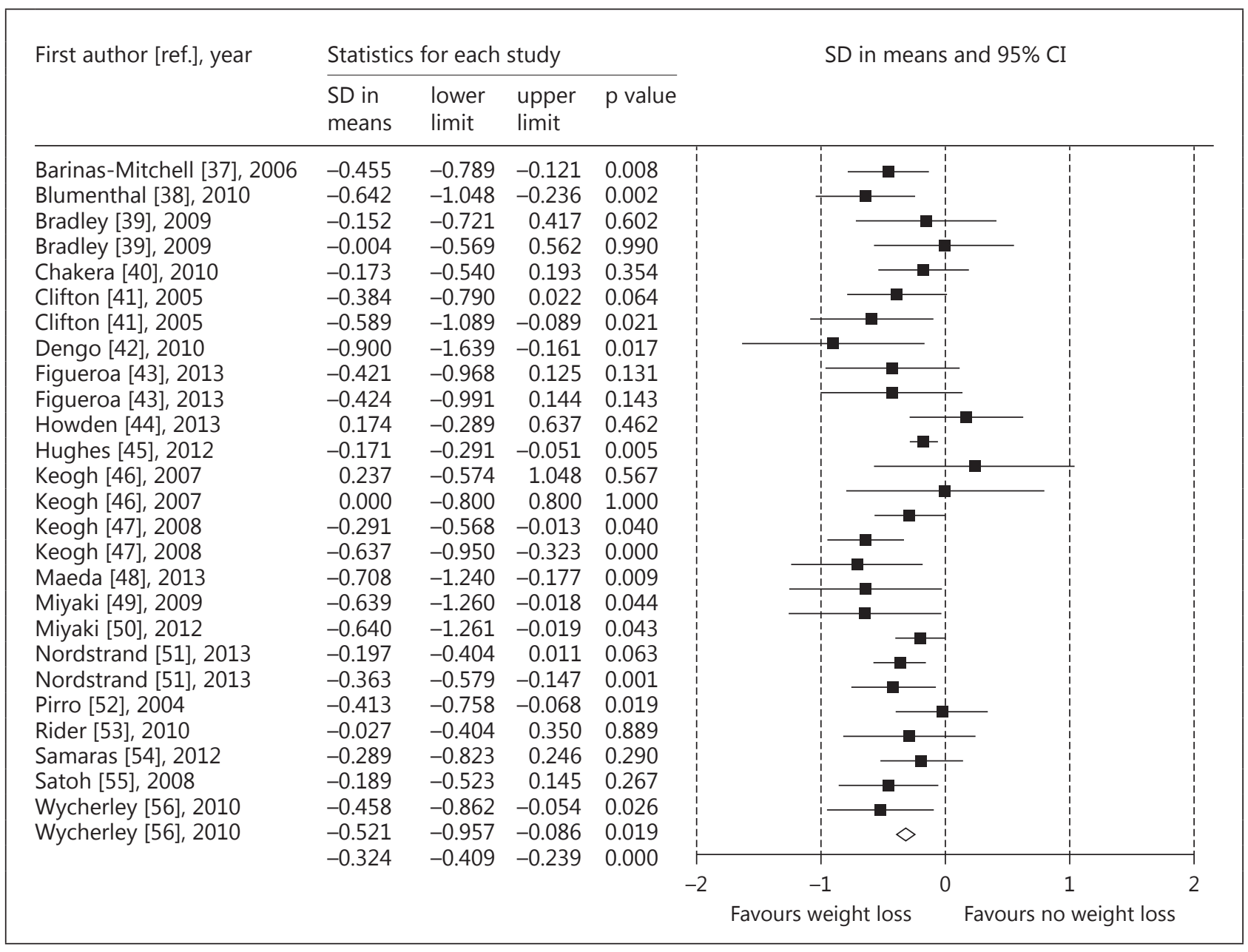

Fig. 1. The effect of weight loss on PWV measured at all arterial segments (adapted from Petersen et al. [5]).

\section{Dietary Intake and PWV}

The results of this meta-analysis suggest that weight loss may help improve vascular function, although improving dietary quality may also be another strategy to improve PWV. In 2011, Pase et al. [13] conducted a systematic review and meta-analysis of randomised controlled trials looking at the effect of dietary and nutrient interventions to improve measures of arterial stiffness (including PWV). It was found that omega 3 and soy isoflavone supplementation improved PWV, and there was limited evidence for a beneficial effect of vitamin and micro-nutrient supplementation. Epidemiological studies conducted in cohorts of healthy, hypertensive or high-risk CVD subjects suggest that the consumption of fruit and vegetables [14, 15], dairy foods [15-17] and flavonoids including soy [18] may be inversely associated with PWV, while the consumption of saturated fat [19] has a detrimental effect. Observational data for the effect of sodium on PWV is mixed. Lee et al. [20] showed an inverse association between predicted 24-hour sodium excretion (Tanaka's equation) and PWV, and García-Ortiz et al. [21] reported a non-significant J-shaped relationship between 24-hour sodium excretion and PWV. Dietary patterns such as the DASH diet [22] and the Mediterranean diet [23] seem to improve PWV; however, nutrient-poor, energy-dense patterns of 
Petersen et al.: Weight Loss, Dietary Intake and Pulse Wave Velocity

intake have a detrimental effect [24]. Intervention studies to support these findings are lacking or inconsistent.

In a randomised controlled trial of subjects at high risk of CVD with a habitually low intake of fruit and vegetables, the consumption of $+2,+4$ and +6 portions of high-flavonoid fruit and vegetables or low-flavonoid fruit and vegetables per day for 6 weeks (above habitual intake) did not affect cfPWV after 18 weeks once the data was adjusted for heart rate [25]. In the study by Macready et al. [25], the number of serves of fruit per day derived from fruit juice was not reported; however, the participants were allowed to include fruit juice, and this may help explain their finding, as it has been shown that fruit juice consumption is positively associated with arterial stiffness because of the high sugar content and lack of fibre [26]. Therefore, further research is required from well-designed randomised controlled trials to determine the effect of fruit and vegetable consumption on PWV and the mechanisms responsible. There is evidence to indicate that polyphenols and nitrates contained in fruit and vegetables may contribute to the beneficial effect on PWV [27]. A high potassium intake reduces PWV in subjects with hypertension in a dose-dependent manner, which may also explain the effect of fruit and vegetables [28].

Intervention studies looking at the effect of sodium on PWV have shown that in normotensive populations, sodium supplementation/a high-sodium diet for 2-6 weeks has no effect on PWV [29-31]. In hypertensive populations, a high-sodium diet ( $>90 \mathrm{mmol} / 24 \mathrm{~h}$ ) compared to a lower sodium intake increases PWV [32-34]. Pimenta et al. [35] observed no reduction in PWV when the sodium intake was reduced to $50 \mathrm{mmol} / 24 \mathrm{~h}$ compared to $250 \mathrm{mmol} / 24 \mathrm{~h}$ for 7 days in a cohort of subjects with resistant hypertension, but blood pressure fell significantly. In summary, in subjects with hypertension, either unmedicated or responsive to antihypertensive therapy, sodium intake is positively associated with arterial stiffness. In normotensive populations, an increase in the sodium intake for up to 6 weeks does not seem to change PWV; however, it is not clear what the long-term effect is.

Epidemiological studies have indicated that a higher consumption of dairy products is associated with less arterial stiffening [15-17]. In the Maine-Syracuse Longitudinal Study, the frequency of the overall dairy consumption was inversely associated with cfPWV [16]. In a study of healthy Spanish subjects, Recio-Rodriguez et al. [17] found that only the consumption of low-fat dairy was associated with a reduced cfPWV, such that per $100 \mathrm{~g} /$ day increase in low-fat dairy, cfPWV was $0.101 \mathrm{~m} / \mathrm{s}$ lower $(95 \% \mathrm{CI}-0.178,-0.023 ; \mathrm{p}=0.011)$. However, consuming whole-fat dairy was associated with a $0.109-\mathrm{m} / \mathrm{s}$ increase $(95 \%$ CI $0.006,0.213)$ in cfPWV per $100 \mathrm{~g} /$ day [17]. There have been limited randomised controlled trials conducted in this area. A recent randomised, cross-over study showed that an additional 4 servings of non-fat dairy per day reduced cfPWV compared with the no-dairy condition (4 servings of fruit juice) [36]. As previously mentioned, fruit juice has been shown to have a detrimental effect on arterial stiffness, and therefore, the result of this study may be a reflection of this rather than the effect of dairy. In summary, data from observational and intervention studies are not consistent with regard to the effect of dietary intake on PWV, and this is largely due to the heterogeneity between the studies and the lack of well-designed randomised controlled trials.

\section{Conclusion}

The most promising dietary strategy for improving PWV seems to be weight loss. However, concomitantly improving the dietary quality and achieving weight loss may improve vascular function to a greater extent. In the future, well-designed randomised controlled trials are required to determine the optimal macro-nutrient composition of weight loss diets 
to reduce PWV, and the mechanisms that explain the effect of weight loss on PWV need to be explored. In addition, greater evidence is required for the effect of individual dietary components and patterns of intake on PWV.

\section{Disclosure Statement}

The authors have no disclosures.

\section{References}

$>1$ Bauer UE, Briss PA, Goodman RA, Bowman BA: Prevention of chronic disease in the 21st century: elimination of the leading preventable causes of premature death and disability in the USA. Lancet 2014;384:45-52.

2 World Health Organization: Global health observatory data: obesity situations and trends. 2015. http://www. who.int/gho/ncd/risk_factors/obesity_text/en/ (accessed April 4, 2015).

-3 Fan J, Song Y, Chen Y, Hui R, et al: Combined effect of obesity and cardio-metabolic abnormality on the risk of cardiovascular disease: a meta-analysis of prospective cohort studies. Int Journal Cardiol 2013;168:47614768.

4 Cohen J, Cohen D: Cardiovascular and renal effects of weight reduction in obesity and the metabolic syndrome. Curr Hypertens Rep 2015;17:1-7.

5 Petersen KS, Blanch N, Keogh JB, Clifton PM: Effect of weight loss on pulse wave velocity: systematic review and meta-analysis. Arterioscler Thromb Vasc Biol 2015;35:243-252.

6 Laurent S, Cockcroft J, Van Bortel L, Boutouyrie P, et al: Expert consensus document on arterial stiffness: methodological issues and clinical applications. Eur Heart J 2006;27:2588-2605.

7 Ben-Shlomo Y, Spears M, Boustred C, May M, et al: Aortic pulse wave velocity improves cardiovascular event prediction: an individual participant meta-analysis of prospective observational data from 17,635 subjects. J Am Coll Cardiol 2014;63:636-646.

-8 Cecelja M, Chowienczyk P: Dissociation of aortic pulse wave velocity with risk factors for cardiovascular disease other than hypertension: a systematic review. Hypertension 2009;54:1328-1336.

-9 Jain S, Khera R, Corrales-Medina VF, Townsend RR, et al: Inflammation and arterial stiffness in humans. Atherosclerosis 2014;237:381-390.

10 Schmidt FM, Weschenfelder J, Sander C, Minkwitz J, et al: Inflammatory cytokines in general and central obesity and modulating effects of physical activity. PLoS One 2015;10:e0121971.

11 Agrawal V, Krause KR, Chengelis DL, Zalesin KC, et al: Relation between degree of weight loss after bariatric surgery and reduction in albuminuria and C-reactive protein. Surg Obes Relat Dis 2009;5:20-26.

12 Booth GL, Kapral MK, Fung K, Tu JV: Relation between age and cardiovascular disease in men and women with diabetes compared with non-diabetic people: a population-based retrospective cohort study. Lancet 2006; 368:29-36.

13 Pase MP, Grima NA, Sarris J: The effects of dietary and nutrient interventions on arterial stiffness: a systematic review. Am J Clin Nutr 2011;93:446-454.

14 Aatola H, Koivistoinen T, Hutri-Kähönen N, Juonala M, et al: Lifetime fruit and vegetable consumption and arterial pulse wave velocity in adulthood/clinical perspective. Circulation 2010;122:2521-2528.

15 Petersen KS, Keogh JB, Meikle PJ, Garg ML, et al: Dietary predictors of arterial stiffness in a cohort with type 1 and type 2 diabetes. Atherosclerosis 2015;238:175-181.

16 Crichton GE, Elias MF, Dore GA, Abhayaratna WP, et al: Relations between dairy food intake and arterial stiffness. Hypertension 2012;59:1044-1051.

17 Recio-Rodriguez J, Gomez-Marcos M, Patino-Alonso M-C, Sanchez A, et al: Association between fat amount of dairy products with pulse wave velocity and carotid intima-media thickness in adults. Nutr J 2014;13:37.

$\rightarrow 18$ Lilamand M, Kelaiditi E, Guyonnet S, Antonelli Incalzi R, et al: Flavonoids and arterial stiffness: promising perspectives. Nutr Metab Cardiovasc Dis 2014;24:698-704.

19 Livingstone KM, Givens DI, Cockcroft JR, Pickering JE, et al: Is fatty acid intake a predictor of arterial stiffness and blood pressure in men? Evidence from the Caerphilly Prospective Study. Nutr Metab Cardiovasc Dis 2013; 23:1079-1085.

20 Lee SK, Kim JS, Kim SH, Kim YH, et al: Sodium excretion and cardiovascular structure and function in the nonhypertensive population: the Korean Genome and Epidemiology Study. Am J Hypertens 2014, Epub ahead of print.

-21 García-Ortiz L, Recio-Rodríguez JI, Rodríguez-Sánchez E, Patino-Alonso MC, et al: Sodium and potassium intake present a J-shaped relationship with arterial stiffness and carotid intima-media thickness. Atherosclerosis 2012;225:497-503.

22 Al-Solaiman Y, Jesri A, Zhao Y, Morrow JD, et al: Low-sodium DASH reduces oxidative stress and improves vascular function in salt-sensitive humans. J Hum Hypertens 2009;23:826-835. 
Petersen et al.: Weight Loss, Dietary Intake and Pulse Wave Velocity

23 van de Laar RJJ, Stehouwer CDA, van Bussel BCT, Prins MH, et al: Adherence to a Mediterranean dietary pattern in early life is associated with lower arterial stiffness in adulthood: the Amsterdam Growth and Health Longitudinal Study. J Intern Med 2013;273:79-93.

24 Kesse-Guyot E, Vergnaud A-C, Fezeu L, Zureik M, et al: Associations between dietary patterns and arterial stiffness, carotid artery intima-media thickness and atherosclerosis. Eur J Cardiovasc Prev Rehabil 2010;17: 718-724.

25 Macready AL, George TW, Chong MF, Alimbetov DS, et al: Flavonoid-rich fruit and vegetables improve microvascular reactivity and inflammatory status in men at risk of cardiovascular disease - FLAVURS: a randomized controlled trial. Am J Clin Nutr 2014;99:479-489.

26 Pase MP, Grima N, Cockerell R, Pipingas A: Habitual intake of fruit juice predicts central blood pressure. Appetite 2015;84:68-72.

27 Kapil V, Khambata RS, Robertson A, Caulfield MJ, et al: Dietary nitrate provides sustained blood pressure lowering in hypertensive patients a randomized, phase 2, double-blind, placebo-controlled study. Hypertension 2015;65:320-327.

28 He FJ, Marciniak M, Carney C, Markandu ND, et al: Effects of potassium chloride and potassium bicarbonate on endothelial function, cardiovascular risk factors, and bone turnover in mild hypertensives. Hypertension 2010;55:681-688.

29 Dickinson KM, Keogh JB, Clifton PM: Effects of a low-salt diet on flow-mediated dilatation in humans. Am J Clin Nutr 2009;89:485-490.

30 Dickinson KM, Clifton PM, Keogh JB: A reduction of $3 \mathrm{~g} /$ day from a usual $9 \mathrm{~g} /$ day salt diet improves endothelial function and decreases endothelin-1 in a randomised cross_over study in normotensive overweight and obese subjects. Atherosclerosis 2014;233:32-38.

-31 Todd AS, Macginley RJ, Schollum JB, Williams SM, et al: Dietary sodium loading in normotensive healthy volunteers does not increase arterial vascular reactivity or blood pressure. Nephrology 2012;17:249-256.

32 He FJ, Marciniak M, Visagie E, Markandu ND, et al: Effect of modest salt reduction on blood pressure, urinary albumin, and pulse wave velocity in White, Black, and Asian mild hypertensives. Hypertension 2009;54:482488.

33 Todd AS, MacGinley RJ, Schollum JB, Johnson RJ, et al: Dietary salt loading impairs arterial vascular reactivity. Am J Clin Nutr 2010;91:557-564.

-34 Seals DR, Tanaka H, Clevenger CM, Monahan KD, et al: Blood pressure reductions with exercise and sodium restriction in postmenopausal women with elevated systolic pressure: role of arterial stiffness. J Am Coll Cardiol 2001;38:506-513.

-35 Pimenta E, Gaddam KK, Oparil S, Aban I, et al: Effects of dietary sodium reduction on blood pressure in subjects with resistant hypertension: results from a randomized trial. Hypertension 2009;54:475-481.

-36 Machin DR, Park W, Alkatan M, Mouton M, et al: Effects of non-fat dairy products added to the routine diet on vascular function: a randomized controlled crossover trial. Nutr Metab Cardiovasc Dis 2015;25:364-369.

-37 Barinas-Mitchell E, Kuller LH, Sutton-Tyrrell K, Hegazi R, et al: Effect of weight loss and nutritional intervention on arterial stiffness in type 2 diabetes. Diabetes Care 2006;29:2218-2222.

-38 Blumenthal JA, Babyak MA, Hinderliter A, Watkins LL, et al: Effects of the DASH diet alone and in combination with exercise and weight loss on blood pressure and cardiovascular biomarkers in men and women with high blood pressure: the ENCORE study. Arch Intern Med 2010;170:126-135.

-39 Bradley U, Spence M, Courtney CH, McKinley MC, et al: Low-fat versus low-carbohydrate weight reduction diets: effects on weight loss, insulin resistance, and cardiovascular risk: a randomized control trial. Diabetes 2009;58:2741-2748.

40 Chakera A, Bunce S, Heppenstall C, Smith JC: The effects of weight loss using dietary manipulation and rimonabant therapy on arterial stiffness in type 2 diabetes. Artery Res 2010;4:47-51.

41 Clifton PM, Keogh JB, Foster PR, Noakes M: Effect of weight loss on inflammatory and endothelial markers and FMD using two low-fat diets. Int J Obes (Lond) 2005;29:1445-1451.

-42 Dengo AL, Dennis EA, Orr JS, Marinik EL, et al: Arterial destiffening with weight loss in overweight and obese middle-aged and older adults. Hypertension 2010;55:855-861.

43 Figueroa A, Vicil F, Sanchez-Gonzalez MA, Wong A, et al: Effects of diet and/or low-intensity resistance exercise training on arterial stiffness, adiposity, and lean mass in obese postmenopausal women. Am J Hypertens 2013; 26:416-423.

44 Howden EJ, Leano R, Petchey W, Coombes JS, et al: Effects of exercise and lifestyle intervention on cardiovascular function in CKD. Clin J Am Soc Nephrol 2013;8:1494-1501.

45 Hughes TM, Althouse AD, Niemczyk NA, Hawkins MS, et al: Effects of weight loss and insulin reduction on arterial stiffness in the SAVE trial. Cardiovasc Diabetol 2012;11:114.

$\checkmark 46$ Keogh JB, Brinkworth GD, Clifton PM: Effects of weight loss on a low-carbohydrate diet on flow-mediated dilatation, adhesion molecules and adiponectin. Br J Nutr 2007;98:852-859.

47 Keogh JB, Brinkworth GD, Noakes M, Belobrajdic DP, et al: Effects of weight loss from a very-low-carbohydrate diet on endothelial function and markers of cardiovascular disease risk in subjects with abdominal obesity. Am J Clin Nutr 2008;87:567-576.

48 Maeda S, Miyaki A, Kumagai H, Eto M, et al: Lifestyle modification decreases arterial stiffness and plasma asymmetric dimethylarginine level in overweight and obese men. Coron Artery Dis 2013;24:583-588. 
49 Miyaki A, Maeda S, Yoshizawa M, Misono M, et al: Effect of weight reduction with dietary intervention on arterial distensibility and endothelial function in obese men. Angiology 2009;60:351-357.

50 Miyaki A, Maeda S, Choi Y, Akazawa N, et al: The addition of whole-body vibration to a lifestyle modification on arterial stiffness in overweight and obese women. Artery Res 2012;6:85-91.

51 Nordstrand N, Gjevestad E, Hertel JK, Johnson LK, et al: Arterial stiffness, lifestyle intervention and a lowcalorie diet in morbidly obese patients - a nonrandomized clinical trial. Obesity (Silver Spring) 2013;21: 690-697.

52 Pirro M, Schillaci G, Savarese G, Gemelli F, et al: Attenuation of inflammation with short-term dietary intervention is associated with a reduction of arterial stiffness in subjects with hypercholesterolaemia. Eur J Cardiovasc Prev Rehabil 2004;11:497-502.

-53 Rider OJ, Tayal U, Francis JM, Ali MK, et al: The effect of obesity and weight loss on aortic pulse wave velocity as assessed by magnetic resonance imaging. Obesity 2010;18:2311-2316.

54 Samaras K, Viardot A, Lee PN, Jenkins A, et al: Reduced arterial stiffness after weight loss in obese type 2 diabetes and impaired glucose tolerance: the role of immune cell activation and insulin resistance. Diab Vasc Dis Res 2013;10:40-48.

55 Satoh N, Shimatsu A, Kato Y, Araki R, et al: Evaluation of the cardio-ankle vascular index, a new indicator of arterial stiffness independent of blood pressure, in obesity and metabolic syndrome. Hypertens Res 2008;31: 1921-1930.

56 Wycherley TP, Brinkworth GD, Keogh JB, Noakes M, et al: Long-term effects of weight loss with a very low carbohydrate and low fat diet on vascular function in overweight and obese patients. J Intern Med 2010;267: 452-461. 